Ann. Génét. Sél. anim., I974, 6 (I), 9I-I02.

\title{
L'HÉRITABILITÉ DU NOMBRE DE MAMELLES \\ CHEZ LE PORC DE PIETRAIN ET LE PORC LANDRACE BELGE
}

\author{
R. HANSET et R. CAMERLYNCK* \\ Faculté de Médecine Vétérinaire, \\ Université de Liège, \\ 45, rue des Vétérinaires, \\ Cureghem, Bruxelles \\ * Service de l'Élevage, \\ Ministère de l'Agriculture, \\ Bruxelles \\ RÉSUMÉ
}

Sur deux échantillons composés de 56 verrats, 560 truies et 4342 porcelets de la race de Piétrain et 45 verrats, 197 truies et I 579 porcelets de la race Landrace belge, les auteurs ont calculé :

Io le nombre moyen de tétines, l'écart-type, la statistique $g_{1}$ du test de symétrie ;

$2^{\circ}$ l'héritabilité du nombre de tétines, par la méthode d'analyse de variance et par la méthode de la régression des descendants sur les parents ;

$3^{\circ}$ la régression du nombre de porcelets vivants à la naissance et à 3 semaines sur le nombre de tétines de la mère.

Chez le porc de Piétrain, le nombre moyen de tétines est de 13,I7 contre 13,99 chez le porc Landrace. En outre, chez le porc de Piétrain, la distribution est asymétrique, avec excès de valeurs supérieures à la moyenne. Les différentes estimations de l'héritabilité oscillent autour de la valeur de 0,46 chez le porc de Piétrain et autour de la valeur de 0,34 chez le porc Landrace. La corrélation positive entre le nombre de tétines de la mère et le nombre de porcelets vivants est très faible et non significative.

\section{INTRODUCTION}

Le nombre de mamelles, évalué par le nombre de tétines, est dans l'espèce porcine, un caractère variable. Déterminé à la naissance, ce caractère quantitatif échappe aux influences du milieu externe auxquelles sont si sensibles des caractères de pro- 
duction tels que la croissance, la production laitière, etc. Aucune correction pour des facteurs de milieu n'étant nécessaire, les données récoltées sur le terrain se prêtent comme telles à l'analyse génétique.

NACHTSheim (I925 $a$ et $b$ ) distingue, chez le porc, trois types de tétines : I) les tétines normales, au nombre de 7 paires; 2) les tétines surnuméraires, asymériques dont la position la plus fréquente est entre la $3^{\mathrm{e}}$ et $\mathrm{la} 4^{\mathrm{e}}$ paire de tétines normales plus rarement entre la $4^{\mathrm{e}}$ et la $5^{\mathrm{e}}$ paire, plus rarement encore entre d'autres paires de tétines normales ; 3) les tétines rudimentaires à situation postérieure chez la truie, sur le sac scrotal chez le verrat.

On suspecte, depuis longtemps déjà, que les variations du nombre de tétines chez le porc ont un substrat génétique. Dès I9I3, WENTWORTH entreprend l'étude du déterminisme génétique de ces variations. Pour WENTworth, 1'absence de tétines rudimentaires domine la présence et est due à un gène lié au sexe ; les variations du nombre de tétines normales ne peuvent s'expliquer par un modèle génétique simple.

NACHTSHEIM (I925) ne peut confirmer l'observation de WENTWORTH, relative à l'hérédité des tétines rudimentaires. En ce qui concerne les tétines normales, il conclut à l'intervention de plusieurs facteurs héréditaires. Comme nous l'avons vu, pour NACHTSHEIm, le nombre de base de tétines normales est de 7 paires et la variation porte sur les paires $n^{\circ} 2$ et $n^{\circ} 6,1^{\prime}$ absence de cette dernière étant plus fréquente que celle de la paire no 2 .

Chez le sanglier européen, les paires $n^{\circ} 2$ et $n^{\circ} 6$ manquent régulièrement. On se demande, dès lors, d'où viennent les paires 2 et 6 communément présentes che $z$ le porc domestique. NACHTSHEIM fait intervenir Sus vittatus dans la constitution de nos porcs domestiques. Un porc chinois, qui dérive de Sus vittatus, le Maskenschwein, présente un appareil mammaire fort développé à quatorze mamelles, plus une ou deux paires supplémentaires, soit un total de huit à neuf paires.

Il faut attendre r959 pour voir appliquer les méthodes de la génétique quantitative à l'étude des variations du nombre de tétines chez le porc (ALLEN et al., I959).

L'intérêt porté au nombre de mamelles s'explique si on pense qu'il pourrait exister une relation entre ce caractère et la prolificité de la souche. Dès I9I3, PEARI, d'une part, PARKER et BULLARD d'autre part s'interrogent sur cette relation éventuelle et la confrontent avec les faits.

Les objets de notre étude seront les suivants :

$I^{\circ}$ comparer les deux races envisagées pour la distribution du nombre de tétines; $2^{\circ}$ comparer, à l'intérieur de chacune des races, différentes méthodes d'estimation de l'héritabilité du caractère ;

$3^{\circ}$ comparer les deux races du point de vue de leur variabilité génétique ;

$4^{\circ}$ étudier la relation entre le nombre de tétines d'une part, la prolificité et la viabilité des porcelets d'autre part.

\section{MATÉRIEL ET MÉTHODES}

Nous avons pu disposer des données relatives au nombre de porcelets nés vivants, au nombre de porcelets vivants au marquage ( \pm 3 semaines), au nombre de tétines des porcelets vivants au marquage pour : I) en race de Piétrain : 560 nichées (4 342 porcelets) issues de 560 truies et de 
56 verrats ; 2) en race Landrace belge : 197 nichées (I 579 porcelets) issues de I97 truies et de 45 verrats. Il s'agit de portées nées au cours de l'année r96i, dans la province de Brabant (le relevé de ces données fait partie du travail de routine des contrôleurs de naissance des syndicats d'élevage).

Le coefficient d'héritabilité du nombre de tétines à été estimé à partir :

I. de la composante paternelle obtenue de l'analyse de variance après classification hiérarchique : " entre verrats" - " entre truies intra-verrat " - " entre pleins-frères";

2. de la composante maternelle obtenue dans la même analyse de variance qu'en I) ;

3. des composantes paternelle et maternelle obtenues ci-dessus;

4. de la régression de la moyenne des descendants sur la mère, intra-père ;

5. même chose qu'en 4), avec, en outre, pondération pour les nombres différents de descendants par portée, selon KEMPThorne et TANDON (I953), REeve (I955), Falconer (I963);

6. de la régression de la moyenne des descendants d'une portée sur le père, un père donné apparaissant ainsi autant de fois qu'il a de portées;

7. même chose qu'en 6), avec, en outre, pondération pour les nombres différents de descendants par portée ;

8. régression de la moyenne des descendants d'une portée, sur la moyenne parentale ;

9. même chose qu'en 8), avec, en outre, pondération pour les nombres différents de descendants.

\section{RÉSULTATS}

Les données relatives à la distribution du nombre de tétines sont données au tableau I, pour le porc de Piétrain, au tableau 2 pour le porc Landrace belge.

Il y a une nette différence entre les moyennes des deux races, le porc Landrace belge ayant, en moyenne, 13,99 tétines (intervalle de variation I0-18), le porc de Piétrain ayant, en moyenne, I3,I7 tétines (intervalle de variation I0-I6). Néanmoins, la valeur modale est de I4, pour les deux races. La variance est systématiquement plus élevée chez le porc Landrace que chez le porc de Piétrain. En outre, la distribution est asymétrique chez le porc de Piétrain (excès de valeurs plus grandes que la moyenne). Il n'y a pas de différence significative entre sexes, dans l'une et l'autre race.

On notera que 6,49 p. Ioo d'individus chez le porc de Piétrain et 0,38 p. Ioo chez le porc Landrace n'atteignent pas le minimum requis de douze tétines. Le nombre moyen de tétines étant plus proche de ce minimum pour le porc de Piétrain que pour le porc Landrace, la sélection exercée chez les parents y est plus forte. Cela se traduit par une différence entre la moyenne parentale et la moyenne des descendants, plus importante chez le porc de Piétrain. Il en est de même si on compare les variances des parents et des descendants.

Les neuf estimations du coefficient d'héritabilité sont données au tableau 3 , pour les deux races. Pour le porc de Piétrain ces estimations oscillent entre 0,352 et 0,566 ; pour le porc Landrace entre 0,189 et 0,477 . Ces coefficients ont tendance à être plus élevés pour le porc de Piétrain.

A partir des composantes de la variance, paternelle et maternelle, on peut estimer le terme $c^{2}$, composante "nichée » de la variance totale : il est égal à 0,0345 chez le porc de Piétrain et à $0,036 \mathrm{chez}$ le Landrace.

La covariance père-enfant peut être différente de la covariance mère-enfant, cette dernière pouvant contenir un composant non génétique. Nous observons au tableau 3 que la régression des descendants sur la mère est tantôt plus grande ( $\mathrm{Lan}$ drace) tantôt plus petite (Piétrain) que la régression sur le père. 
TABLEAU I

Distribution du nombre de tétines chez le porc de Piétrain

\begin{tabular}{|c|c|c|c|c|c|}
\hline \multirow{3}{*}{ Nombre de tétines } & \multicolumn{5}{|c|}{ Fréquences } \\
\hline & \multirow[b]{2}{*}{ Pères } & \multirow[b]{2}{*}{ Mères } & \multicolumn{3}{|c|}{ Porcelets } \\
\hline & & & Mâles & Femelles & $\begin{array}{c}\text { Mâles } \\
+\quad \text { femelles }\end{array}$ \\
\hline 10 & & & 27 & 48 & 75 \\
\hline 11 & & & 105 & 102 & 207 \\
\hline 12 & 11 & 107 & 362 & 441 & 803 \\
\hline 13 & 14 & 166 & 528 & 646 & 1174 \\
\hline 14 & 30 & 276 & 926 & 1051 & 1977 \\
\hline 15 & 1 & 11 & 49 & 45 & 94 \\
\hline 16 & & & 8 & 4 & 12 \\
\hline Totaux & 56 & 560 & 2005 & 2337 & 4342 \\
\hline Moyenne & 13,37 & 13,34 & 13,20 & 13,16 & 13,17 \\
\hline Écart-type & 0,82 & 0,80 & 1,03 & 1,03 & 1,03 \\
\hline Coefficient variation & 6,14 & 6,03 & 7,83 & 7,85 & 7,84 \\
\hline Test de symétrie $(t)$ & $-1,88$ & $-4,52 * * *$ & $-13,88^{* * *}$ & $-16,95 * * *$ & $-21,84 * * *$ \\
\hline
\end{tabular}

*** : significatif au seuil 1. p. 1000.

TABLEAU 2

Distribution du nombre de tétines chez le porc Landrace belge

\begin{tabular}{|c|c|c|c|c|c|}
\hline \multirow{3}{*}{ Nombre de tétines } & \multicolumn{5}{|c|}{ Fréquences } \\
\hline & \multirow[b]{2}{*}{ Pères } & \multirow[b]{2}{*}{ Meres } & \multicolumn{3}{|c|}{ Porcelets } \\
\hline & & & Mâles & Femelles & $\begin{array}{c}\text { Mâles } \\
+ \text { femelles }\end{array}$ \\
\hline 10 & & & & 1 & 1 \\
\hline 11 & & & 3 & 2 & 5 \\
\hline 12 & 2 & 11 & 81 & 107 & 188 \\
\hline 13 & 8 & 23 & 69 & 81 & 150 \\
\hline 14 & 28 & 115 & 369 & 516 & 885 \\
\hline 15 & 2 & 25 & 72 & 103 & 175 \\
\hline 16 & 4 & 23 & 66 & 98 & 164 \\
\hline 17 & 1 & & 2 & 6 & 8 \\
\hline 18 & & & 1 & 2 & 3 \\
\hline Totaux & 45 & 197 & 663 & 916 & 1579 \\
\hline Moyenne & 14,02 & 14,13 & 13,96 & 14,02 & 13,99 \\
\hline Écart-type & 0,99 & 0,96 & 1,09 & 1,11 & 1,10 \\
\hline Coefficient variation & 7,05 & 6,79 & 7,82 & 7,90 & 7,87 \\
\hline Test de symétrie $(t)$ & 2,38 & 0,88 & 0,16 & 0,48 & 0,49 \\
\hline
\end{tabular}


TABLEAU 3

Estimations du coefficient d'héritabilité $\left(h^{2}\right)$ et de son écart-type $\left(s\left(h^{2}\right)\right)$

\begin{tabular}{|c|c|c|}
\hline \multirow{2}{*}{ Méthode } & \multicolumn{2}{|c|}{ Race } \\
\hline & Piétrain & Landrace \\
\hline 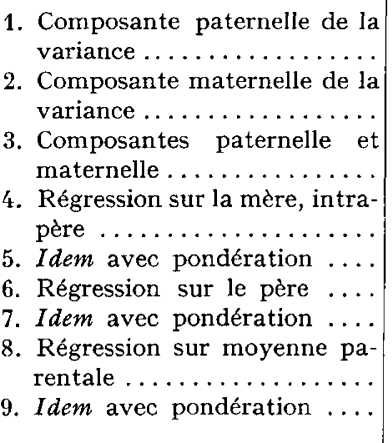 & $\begin{array}{l}0,428 \pm 0,099 \\
0,566 \pm 0,060 \\
0,497 \pm 0,056 \\
0,352 \pm 0,056 \\
0,355 \pm 0,031 \\
0,543 \pm 0,060 \\
0,538 \pm 0,048 \\
0,444 \pm 0,038 \\
0,443 \pm 0,022\end{array}$ & $\begin{array}{l}0,331 \pm 0,114 \\
0,477 \pm 0,101 \\
0,404 \pm 0,065 \\
0,437 \pm 0,092 \\
0,397 \pm 0,040 \\
0,189 \pm 0,090 \\
0,180 \pm 0,033 \\
0,329 \pm 0,061 \\
0,316 \pm 0,032\end{array}$ \\
\hline
\end{tabular}

Dans l'estimation des trois types de régression, on a pris, comme variable dépendante $(y)$ la moyenne des descendants et, comme variable indépendante $(x)$ la valeur parentale : (mère, père, moyenne des deux parents). Cette façon de procéder donne trop peu de poids aux grandes familles. Si nous avions pris chaque descendant séparément et compté chaque parent autant de fois qu'il a de descendants, nous aurions donné trop de poids aux grandes familles. Une méthode a été utilisée qui réalise une pondération pour la taille des familles. On constate (tabl. 3) qu'avec le type de matériel utilisé, il n'y a guère de différence entre les estimations de $h^{2}$, selon que l'on effectue la pondération ou non. Par contre, l'écart-type des estimations avec pondération, calculé selon la méthode proposée par FALCONER (I963), est sensiblement plus petit.

Le problème de la relation entre le nombre de tétines des mères et le nombre de porcelets vivants, soit à la naissance, soit au marquage, est étudié dans les tableaux 4 et 5 .

\section{TABLEAU 4}

Relation (coefficient de régression) entre le nombre de tétines des mères et le nombre de porcelets vivants à la naissance et au marquage

\begin{tabular}{|c|c|c|}
\hline Race & Piétrain & Landrace belge \\
\hline Porcelets nés vivants & $+0,025 \_0,108$ & $+0,102 \pm 0,163$ \\
\hline $\begin{array}{l}\text { Porcelets vivants } \\
\text { au marquage }\end{array}$ & $+0,118 \pm 0,112$ & $+0,111 \pm 0,142$ \\
\hline
\end{tabular}




\section{TABLEAU 5}

Proportion de nichées contenant plus de porcelets (soit à la naissance, soit au marquage) que la mère n'a de tétines

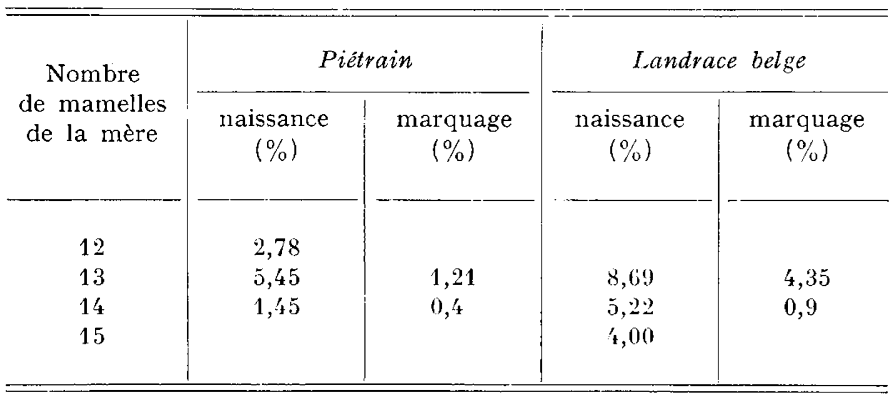

TABLEAU 6

Comparaisons entre races pour le nombre de tétines

\begin{tabular}{|c|c|c|c|c|c|c|}
\hline Races & Effectif & Moyenne & $\begin{array}{l}\text { Écart- } \\
\text { type }\end{array}$ & $\begin{array}{l}\text { Valeur } \\
\text { modale }\end{array}$ & $\begin{array}{c}\text { Test de } \\
\text { symétrie }(t)\end{array}$ & Auteurs \\
\hline Duroc-Jersey ..... & 509 & 11,07 & 1,09 & 10 & $6,28 * * *$ & WENTWORTH, 1913 \\
\hline Duroc-Jersey ..... & 1004 & 12,0 & & & & $\begin{array}{l}\text { Ahlswede } \\
\text { et Robison, } 1965\end{array}$ \\
\hline Landrace (U. S. A.). & 199 & 13,68 & 0,78 & 14 & $8,28 * * *(1)$ & Allen et al., 1959 \\
\hline Landrace (N.). . . . . & 2180 & 14,22 & 0,96 & 14 & $4,47 * * *(2)$ & SKJERVOLd, 1963 \\
\hline Landrace belge..... & 1579 & 13,99 & 1,10 & 14 & 0,49 & Présente étude \\
\hline Large White ..... & 1362 & 14,43 & 0,89 & 14 & $4,47 * * *(2)$ & SKJERVOLD, 1963 \\
\hline Minnesota no $1 \ldots$ & 3565 & 13,50 & 0,84 & 14 & $-16,29^{* * *}$ & $\begin{array}{c}\text { ENFIELD } \\
\text { et REMPEL, } 1961\end{array}$ \\
\hline Piétrain $\ldots . . \ldots$ & 4342 & 13,17 & 1,03 & 14 & $-21,84 * * *$ & Présente étude \\
\hline Poland-china...... & 154 & 12,53 & 1,03 & 12 & $-2,59^{* *}(\mathbf{1})$ & Allen et al., 1959 \\
\hline Yorkshive ........ & 1380 & 13,1 & & & & $\begin{array}{l}\text { Ahlswede } \\
\text { et Robison, } 1965\end{array}$ \\
\hline
\end{tabular}

** : signification au seuil 1 p. 100 ;

*** : signification au seuil 1 p. 1000.

(1) Test calculé d'après l'histogramme publié par les auteurs.

$\left.{ }^{2}\right)$ Test calculé d'après l'histogramme publié par l'auteur et se rapportant globalement aux races Landrace et Large White et aux produits de leur croisement. 
Dans les deux races, on observe une relation positive entre le nombre de tétines des mères et le nombre de porcelets vivants, aux deux époques considérées. Toutefois, le coefficient de régression est faible et non significatif (tabl. 4).

Comme on peut le voir au tableau 5, la proportion de nichées où le nombre de porcelets vivants est supérieur au nombre de tétines des mères est relativement faible. Cette proportion n'est pas nécessairement la plus élevée lorsque le nombre de tétines est le plus petit. Il va de soi que le nombre de tétines fonctionnelles importe plus que le nombre total de tétines.

\section{DISCUSSION}

Des différences raciales assez importantes peuvent exister, en ce qui concerne le nombre moyen de tétines et 1'allure de la distribution (tab1. 6). Les races ayant les plus petits nombres de tétines sont le Duroc-Jersey et le Poland-China; le Large White a le nombre le plus élevé. La distribution du nombre de tétines est souvent asymétrique. Un excès de valeurs supérieures à la moyenne se traduit par une statistique $g_{1}$ négative d'où un test de $t$ négatif, un excès de valeurs inférieures à la moyenne par une statistique $g_{1}$ positive, d'où un test de $t$ positif. Comme on le constate, au tableau 6,1 e signe de l'asymétrie est positif dans certaines races, négatif dans d'autres. A l'exception de la race Duroc-Jersey, l'asymétrie consiste en un excès de valeurs supérieures à la moyenne lorsque la moyenne raciale est inférieure à $\mathrm{I}_{4}$; elle consiste en un excès de valeurs inférieures à la moyenne lorsque la moyenne raciale est supérieure à $\mathrm{I} 4$. Le nombre de $\mathrm{I} 4$ tétines paraît être le nombre charnière pour le porc domestique. Les auteurs n'ont pas observé de différence significative entre les sexes (NAchTSHEIM I925, SKJERVOLD I963).

La plupart des estimations de l'héritabilité relevées dans la littérature sont de l'ordre de 0,3 à 0,4 (tabl. 7).

Les mamelles étant disposées de part et d'autre de la ligne médiane, un coefficient de corrélation (coefficient de répétabilité) peut être calculé entre les nombres de tétines des côtés gauche et droit. Ce coefficient a la signification d'un coefficient d'héritabilité au sens large (il inclut tous les types de variances génétiques), augmenté de certains effets d'environnement. Le tableau 8 fait un relevé des estimations de cette corrélation, trouvées dans la littérature. Les valeurs publiées par PARKER et BULLARD (I9I3) et par NACHTSHEIM (1925) sont plus élevées que celles obtenues par SKJERVOLD (I963) lequel a, parallèlement, estimé l'héritabilité du caractère.

De toutes les méthodes possibles d'estimation de $h^{2}$, ce sont les estimations à partir de la composante maternelle de la variance, de la somme des composantes paternelle et maternelle qui seront les plus proches de l'estimation de la répétabilité. En effet, ce sont ces estimations qui contiennent le plus de termes dus à des effets génétiques non additifs. Les estimations de l'héritabilité et de la répétabilité seront très proches si ces effets sont mineurs.

AlLEN et al. (I959) observent un effet d'hétérosis de 4,47 p. Ioo, le nombre moyen de tétines chez le produit du croisement Landrace $\times$ Poland étant supérieur à la moyenne des parents de 0,57 tétines; ils concluent à l'intervention d'effets de dominance, surdominance ou d'épistasie. Par contre, lors du croisement Landrace 
R. HANSE'T, R. CAMERLYNCK

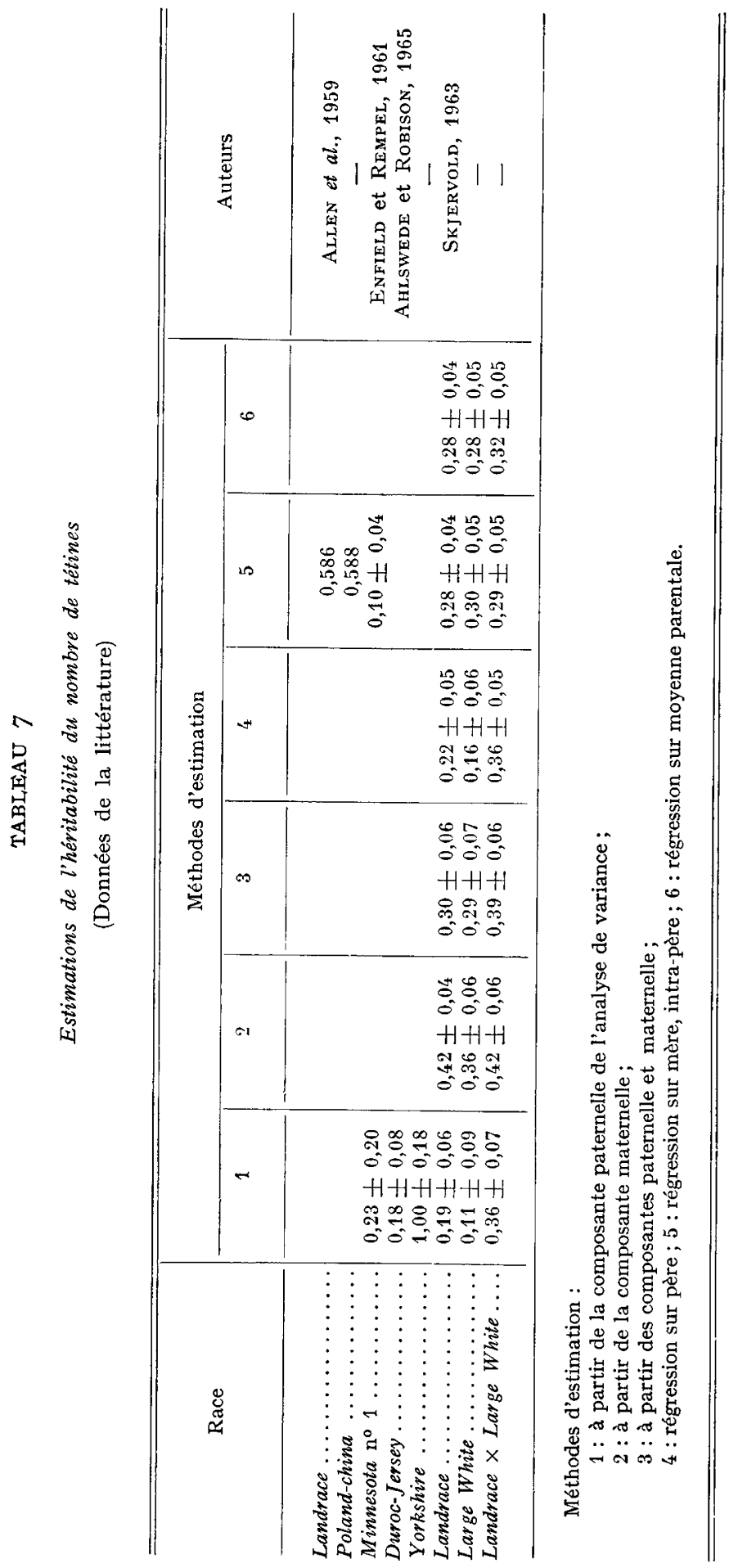




\section{TABLEAU 8}

Corrélation entre le côté gauche et le côté droit pour le nombre de tétines

(Données de la littérature)

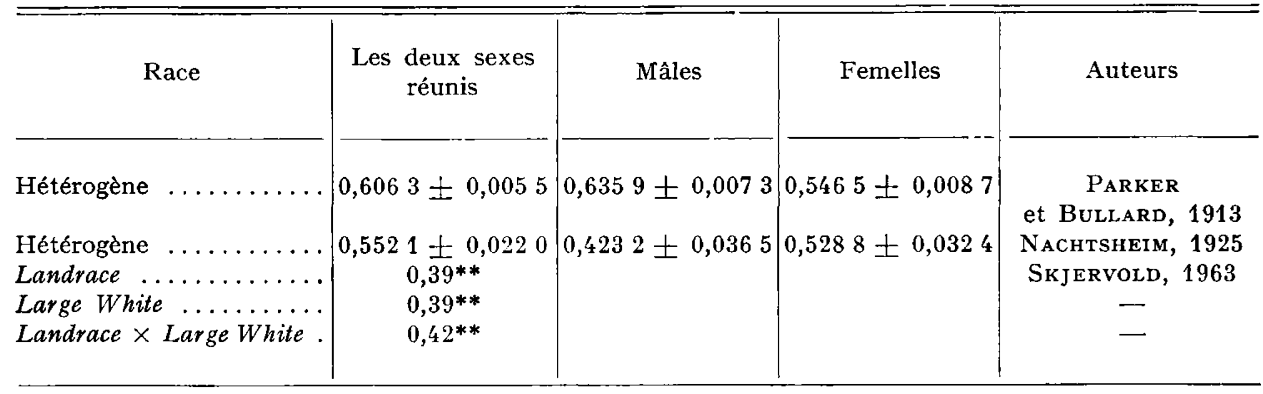

** : significatif au seuil 1 p. 100.

\section{TABLEAU 9}

Corrélations entre le nombre de tétines des mères d'une part, le nombre de porcelets nés vivants, sevrés et le poids de la portée

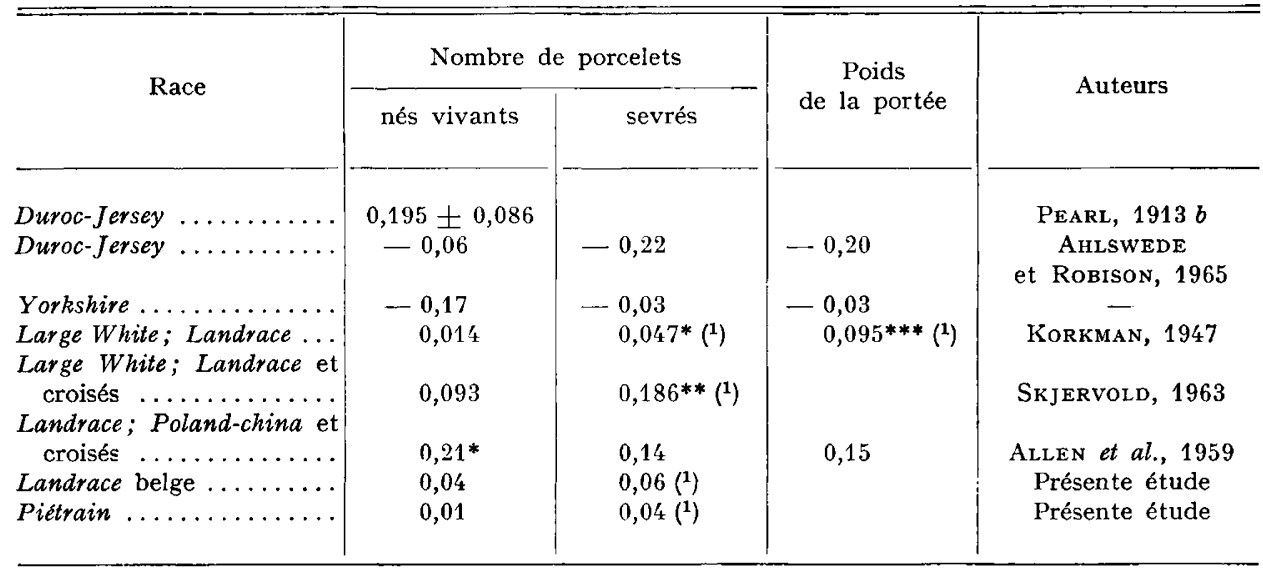

* : significatif au seuil 5 p. 100 ;

** : significatif au seuil 1 p. 100 ;

*** : significatif au seuil 1 p. 1000 .

(1) Calculé à 3 semaines et non au sevrage à 56 jours. 
$\times$ Large White, SkJERvold (I963) constate que le nombre de tétines des croisés est égal à la moyenne des parents.

WILLHAM et WHATLEY (I963) pensent aussi qu'une action génique du type non-additif n'a qu'une importance mineure. En effet, ils n'observent ni déclin dû à la consanguinité, ni hétérosis subséquent.

Les valeurs trouvées dans la littérature pour la corrélation entre le nombre de tétines des mères d'une part, le nombre de porcelets nés vivants, sevrés et le poids de la portée d'autre part sont rapportées au tableau 9. Elles sont très faibles et généralement non significatives.

WILlHAM et WHATLEY (I963) ont aussi étudié l'asymétrie gauche-droite pour le nombre de tétines. L'asymétrie observée leur apparaît, pour la majeure partie, due à des facteurs d'environnement non spécifiés. La corrélation génétique entre le côté gauche et le côté droit est de $+0,97$.

Nous avons observé que la composante maternelle de la variance est supérieure à la composante paternelle. Cette différence peut relever de trois causes : I) effets géniques non-additifs dus à des loci portés par les descendants (dominance, épistasie) ; 2) effets génétiques maternels (loci portés par la mère) ; 3) effets d'environnement communs aux sujets d'une portée (WILLHAM et WHATLEY I963).

WILlHAM et WhATLEY attribuent la faible différence qu'ils observent entre la composante maternelle et la composante paternelle à la seconde des trois éventualités énumérées ci-dessus.

Reçu pour publication en janvier 1974.

\section{REMERCIEMENTS}

Les auteurs tiennent à remercier M. P. Sellier (I. N. R. A.) et J. W. B. King (A. B. R. ().), pour leurs commentaires et suggestions à la lecture du manuscrit.

\section{SUMMARY}

THE HERITABILITY OF THE NUMBER OF TEATS

IN THE PIETRAIN AND BELGIAN LANDRACE PIG BREEDS.

On a sample of 56 boars, 560 sows and 4342 piglets of the Piétrain pig breed and on a sample of 45 boars, I 97 sows and I 579 piglets of the Belgian Landrace breed, the authors have calculated :

I. the mean number of teats, the standard deviation, the statistics $g_{1}$ for the test of symetry ;

2. the heritability of the character, by the methods of variance components analysis and by the methods of regression of offspring on parents;

3. the regression of size of litters on the nipple numbers of the sows.

In the Piétrain pig breed, the average number of nipples is 13,17 but 13,99 in the Belgian Landrace. In the Piétrain pig, the distribution is asymmetrical, with an excess in the number of items larger than the mean.

The average of several estimates of the coefficient of heritability is . 46 for the Piétrain and .34 for the Landrace. The positive correlations found between the nipple number of the sow and litter size at birth and at three weeks are very small and not significant. 


\section{RÉFÉRENCES BIBLIOGRAPHIQUES}

Ahlswede W. T., Robison O. W., 1965. Relationship of nipple number to maternal performance in swine. J. Anim. Sci., 24, 845 .

Allen A. D., Tribble L. F., Lasley J. F., r959. Inheritance of nipple numbers in swine and the relationship to performance. Mo. Agric. Exp. Sta. Res. Bull., 694 .

Burdette W. J., I963. Methodology in Mammalian Genetics. Holden-Day, San-Francisco.

Enfield F. D., Rempel W. E., 196r. Inheritance of teat number and relationship of teat number to various maternel traits in swine. J. Anim. Sci., 20, 876-879.

Falconer D. S., I963. Quantitative Inheritance. Methodology in Mammalian Genetics, 193-216.

Kempthorne O., TAndon D. B., I953. The estimation of heritability by regression of offspring on parent. Biometrics, 9, 90.

Korkman N., 1947. Variation in the size and weight of litters from sows. Acta Agric. Sued., 2, 253-310. NachtSheim H., I925 $a$. Untersuchungen über Variation und Vererbung des Gesäuges beim Scheim I. Z. Tierz. ZiichtBiol., 2, I13-16r.

NAchtsheim H., I925 $b$. Vererbungsversuche an Schweinen : Die Vererbung der Zitzenzahl. $Z$. Induk. Abs. Vererb., 33, 307-3II.

Parker G. H., Bullard C., I9I3. On the size of litters and the number of nipples in swine. Proc. Am. Acad. Arts. Sci., 49, 399-426.

Pearl R., r9r3. On the correlation between the number of mammae of the dam and size of litter in mammals I. Interracial correlation. Proc. Soc. Exp. Biol. Med., 11, 27-30.

Pearl R., I9i3. On the correlation between number of mammae of the dam and size of litter in mammals. II. Intraracial correlation in swine. Proc. Soc. Exp. Biol. Med., 11, 31-32.

ReEve E. C. R., I955. The variance of the genetic correlation coefficient. Biometrics, 11, 357.

SkJERvold H., I963. Inheritance of teat number in swine and the relationship to performance. Acta A gric. Scand., 13, 323-333.

Wentworth E. N., 1913. Inheritance of mamae in Duroc-Jersey swine. Amer. Natur., 47, 257-279 (cité par H. Nachtsheim).

Willham R. L., Whathey J. A., r963. Genetic variation in nipple number in swine. Z. Tierz. Züchtbiol., 78, 350-363. 